\title{
Novel Test Method (Sickle Confirm) to Differentiate Sickle Cell Anemia from Sickle Cell Trait for Potential Use in Developing Countries
}

\author{
TIM R. RANDOLPH, JENNA WHEELHOUSE
}

\begin{abstract}
The objective of this study was to develop a diagnostic testing method to detect $\mathrm{HbS}$, distinguish sickle cell homozygotes from heterozygotes, and overcome testing barriers encountered in laboratories in underdeveloped countries. Blood samples positive and negative for sickle cell were subjected to the standard hemoglobin solubility test followed by a variety of centrifugation and filtration procedures. Each procedure was evaluated for the ability to remove insoluble $\mathrm{HbS}$ from the sample. The hemoglobin types that remain (HbA, $\mathrm{HbA}_{2}$ and $\mathrm{HbF}$ ) were measured spectrophotometrically or estimated visually allowing samples to be categorized into three genotypes (AA, AS and SS) as confirmed by hemoglobin electrophoresis. De-identified EDTA blood samples were obtained from Saint Louis University and Cardinal Glennon Children's hospitals and tested in the Department of Clinical Laboratory Science at Saint Louis University. The main outcome measures were turbidity of the solubility solution; color of the supernatant and the material on the surface of the solution following centrifugation; precipitate trapped on the filter paper; absorbance of the filtrate; and hemoglobin electrophoresis patterns. Centrifugation and filtration successfully separated $\mathrm{HbS}$ from $\mathrm{HbA} / \mathrm{A}_{2} / \mathrm{F}$ allowing for the differentiation of seven sickle cell homozygotes from sixteen heterozygotes with a sensitivity and specificity of $100 \%$. This method has the potential to reliably distinguish homozygous from heterozygous sickle cell patients and it is fast, inexpensive, and simple. These characteristics make Sickle Confirm a desirable method in developing countries like Haiti and Africa where sickle cell anemia is prevalent and modern diagnostic methods like electrophoresis, HPLC and nucleic acid testing are impractical.
\end{abstract}

INDEX TERMS: Sickle Cell Anemia, Hemoglobinopathies, Sickle Cell Disease, Sickle Cell Trait, Third World Countries
Clin Lab Sci 2012;25(1):26

Tim R. Randolph, PhD, MLS, Saint Louis University, St. Louis, $M O$

Jenna Wheelhouse, BS, Boston University, Boston, MA

Address for Correspondence: Tim R. Randolph, PhD, MLS, Saint Louis University, 3437 Caroline Street, St. Louis, MO 63104, (314)977-8518, randoltr@slu.edu

\section{INTRODUCTION}

Sickle cell anemia was first discovered in 1910 by James B. Herrick, a physician practicing in Chicago, who described a 20-year-old black Caribbean dental student as having shortness of breath, palpitations, yellow eyes, and anemia. Dr. Herrick described the patient's blood smear as showing, "thin, sickle-shaped and crescentshaped red cells." Sickle cell anemia was later identified as a genetic disease in which a point mutation in the beta globin gene located on chromosome 11 replaced one original nucleotide, adenine, with thymine. This single nucleotide substitution changes the codon from a GAG to a GTG resulting in the substitution of the amino acid valine for glutamic acid at the sixth position of the beta globin chain producing the sickle hemoglobin (HbS).,3 This genetic mutation produces two distinct genotypes; homozygotes (SS) and heterozygotes (AS), resulting in two distinct clinical syndromes; sickle cell disease (SCD) and sickle cell trait (SCT).

It is estimated that the prevalence of sickle cell trait in Western, Central, and Eastern Africa is between 20 to $40 \%$, while the number of babies with SCD born each year in Africa ranges from 120,000 to $200,000 .{ }^{4}$ Since the people of Haiti are of African descent, the prevalence of sickle hemoglobin is also high at $15.2 \% .^{5}$ 
Patients with SCT exhibit few to no symptoms throughout life except under situations of extreme physiological stress but patients with SCD experience frequent problems that stem from the abnormal intracellular polymerization of the $\mathrm{HbS}$ molecules forming sickle cells. Symptoms of SCD do not present until after six months of age when the gamma to beta switch occurs resulting in the conversion from predominantly $\operatorname{HbF}(2,2)$ to $\operatorname{HbA}(2,2)$, or in the case of sickle cell patients, HbS. Splenic removal of sickle cells causes anemia, splenomegaly and functional hyposplenism. This reduction in spleen function results in frequent infections primarily from Staphylococcus aureus, Streptococcus pneumoniae, and Haemophilus influenza which is the predominant cause of mortality. ${ }^{6}$

The hallmark feature of SCD is acute, painful vasoocclusive crises that can occur anywhere in the body but more commonly occur in the bones, lungs, liver, spleen, penis, eyes, central nervous system (CNS), and urinary tract. The frequency of painful episodes varies from none to as many as six per year. ${ }^{6}$ Infarcts in the brain ranging from silent micro infarcts to acute stroke are a major problem for sickle cell patients. ${ }^{7,8}$

In modern laboratories the diagnosis of patients suspected of having SCD usually involves three levels of testing; CBC to document the severity of the anemia and the presence of sickle cells, hemoglobin solubility screen to identify the presence of $\mathrm{HbS}$, and confirmatory testing involving hemoglobin electrophoresis, high performance liquid chromatography (HPLC), or nucleic acid-based methodologies. Confirmatory testing functions to confirm the presence of $\mathrm{HbS}$, determine zygosity and to document potential concomitant hemoglobinopathies and/or thalassemias.

Treatment protocols for patients with SCD involve prevention of infections with immunizations and prophylactic antibiotic and prevention of crises using hydroxyuria to reactivated the gamma globin gene and transfusions. ${ }^{9}$ During crises patients are given analgesics for pain, and non-steroidal anti-inflammatory drugs administered to manage mild ischemic attacks. Opioids are recommended when pain becomes intolerable or chronic in nature. ${ }^{10}$ The only curative therapies are bone marrow/stem cell transplant and gene transfer therapy. Bone marrow transplantation has proved successful for some individuals but incur risks to include lack of engraftment, life-threatening infections during the period of bone marrow reconstitution and graft vs. host disease (GVHD). Until gene transfer technology allows for the introduction of normally regulated wildtype beta globin genes into erythrocyte precursors, therapy will likely remain focused on the induction of $\mathrm{HbF}$ expression.

\section{Justification for this research study}

Although untreated SCD is a fatal disorder, current treatment protocols can greatly extend the life expectancy of affected individuals. Patients with SCD who live in the developed countries are experiencing longer survival with a reduction in painful crises, lifethreatening infections, and severe vaso-occlusive events, like strokes, due to carefully managed therapeutic interventions. In contrast, underdeveloped countries, where SCD is most prevalent, do not have access to basic medical care and in most cases are without diagnostic and therapeutic interventions needed to manage SCD. For example, laboratory testing to screen for sickle cell is not available in most labs in Haiti and confirmatory testing is nearly non-existent. ${ }^{5}$

The purpose of this study was to develop a novel testing method for differentiating homozygous and heterozygous sickle cell positive patients based on the standard hemoglobin solubility test that is rapid, inexpensive, simple, and reliable. Once a diagnostic testing system is in place, a therapeutic management plan can be developed with the hope of dramatically extending the life expectancy of patients with SCD in developing countries. Such a plan would include the prophylactic administration of clean water, protein supplements, vitamins, prophylactic penicillin, basic immunizations in childhood, and hydroxyurea therapy, in conjunction with reactive/supportive therapy to include activity counseling, exchange transfusions, oxygen, and pain management. Successful diagnosis and treatment of patients can transform SCD in underdeveloped countries from a disease that is fatal in early childhood to a disease that is manageable well into adulthood.

\section{Similar Methods}

A few similar methods were reported in the medical literature in the 1970s and 1980s, but all procedures contain barriers to implementation in underdeveloped countries. Some methods recognized that upon centrifugation, the insoluble $\mathrm{HbS}$ rose to the top of the tube proportionally reducing the red color of the 
solution which was analyzed visually to determine zygosity. ${ }^{11-13}$

In 1983 Bowie and Dohnal developed another modified hemoglobin solubility technique to determine zygosity involving the "SickleQuik" test kit followed by centrifugation and spectrophotometric measurements. The "SickleQuik" method is a biphasic test involving aqueous and organic layers that utilizes the organic solvent toluene. The aqueous phase is identical to the standard hemoglobin solubility test while the organic layer assists in capturing the precipitated hemoglobin. ${ }^{13}$

Lastly, Scabet et al. in 1986 also developed a modified version of the hemoglobin solubility test utilizing filtration and spectrophotometric absorbance measurements to calculate $\mathrm{HbS}$ levels and determine sickle cell zygosity. An equal volume of whole blood collected in EDTA was added to both a hemoglobin solubility tube (saponin, sodium hydrosulfite and a salt solution) and a tube of $\mathrm{dH}_{2} 0$. After the five minute incubation both tubes were filtered through a Whatman \#1 filter paper. Four hundred microliters of each filtrate were added to $2 \mathrm{~mL}$ of cyanmethemoglobin reagent and an absorbance reading was taken on both tubes at $540 \mathrm{~nm}$. The percentage of $\mathrm{HbS}$ was calculated using the formula: $\% \mathrm{HbS}=1$-[Absorbance (sickle tube)/Absorbance (total Hb Tube)]. Heterozygotes had as much as $45 \% \mathrm{HbS}$ while homozygotes had maximum $\mathrm{HbS}$ concentration of $98 \% .^{14}$

All methods previously described had limitations to being implemented in laboratories in underdeveloped countries. None of the methods standardized the blood inoculum introduced into the test system creating false positive and false negative results. If the same volume of blood is added to the test system, a normal sample with moderate anemia would create a pale supernatant or filtrate that would be misdiagnosed as $\mathrm{HbAS}$ and a severely normal anemic sample would create a faint supernatant or filtrate color and be misdiagnosed as HbSS. Other methods required the use of chemical hazards like toluene and cyanide which are carcinogenic and toxic. Developing countries do not have medical waste management systems and most do not have access to trash pick-up and disposal systems. As a result, chemical toxins and carcinogens present a health hazard to developing countries should the chemicals contaminate farmland or enter the water table.
The Scabet method was the most promising to translate into a developing country, but there were still a few barriers to overcome. First, essentially two analyses are performed on each patient sample, one for the analysis and one to determine total hemoglobin in the sample, which doubles the material used, the analysis time, and the confusion. Second, cyanmethemoglobin is added to both tubes for each patient, which adds reagent cost, requires procurement of reagents not readily available in developing countries, and introduces cyanide into an environment where waste disposal is non-existent. Third, a calculation is required to obtain a result for each patient sample introducing the potential for confusion and error in countries where laboratory education is rare and poor. Last, guidelines were not given regarding the absolute cut points necessary to assign a genotype to each sample nor an algorithm to guide clinical decisions when samples fall outside the cut points.

\section{MATERIALS AND METHODS}

Materials

1. Standard hemoglobin solubility test kits SickleStat (Chembio Diagnostics - Medford, NY).

2. Perkin-Elmer Spec Junior 35 uv-vis spectrophotometer

3. FisherBrand Filter Paper - P2, P5 and P8 (Fisher Scientific Company - Suwanee, GA)

4. QuickGel Electrophoresis System (Helena laboratories, Beaumont, TX)

\section{Patient Samples}

Blood samples known to be positive and negative for sickle cell were obtained from the clinical laboratory at Cardinal Glennon Children's Hospital (CGCH) and Saint Louis University Hospital. The blood samples were de-identified by laboratory staff to qualify the study for a waver of IRB oversight.

\section{Methods}

\section{Standardizing the blood inoculum}

Since patients being tested for SCD will span a wide range of hemoglobin levels and the primary endpoint of the analysis will be a spectrophotometric measurement or visual estimation of hemoglobin concentration, we chose to standardize the initial volume of blood introduced into the test system to overcome this obstacle. Normal packed RBCs were mixed with increasing amounts of autologous plasma to create a 
series of samples with increasing hematocrit percentages in increments of $5 \%$, ranging from $15 \%$ to $60 \%$. We selected a target hematocrit of $35 \%$ to represent the average hematocrit obtained among Haitian patients screened by the author. Exactly $50 \mu \mathrm{L}$ (the volume used in the hemoglobin solubility test - HST) of the 35\% hematocrit tube was added to $\mathrm{dH}_{2} \mathrm{O}$ and the absorbance was measured. This became the target absorbance that the other blood dilutions were required to match. The appropriate blood volume was determined that yielded the same target absorbance as the $35 \%$ hematocrit tube produced. The procedure was repeated three times to ensure reproducibility. The data appeared linear so a line of best fit was drawn through the data and a table was constructed to determine the appropriate blood volume based on the hematocrit value (Figure 1 and Table 1) in order to standardize the inoculum of sample added to the test system.

\section{Sickle Confirm Test Method}

The Chembio kit (SickleStat) includes 48 stoppered glass tubes, each containing a pre-weighed amount of sodium hydrosulfite with a blue fill line marked near the top of each tube. The kit also contains a bottle of inorganic buffered salt solution with Saponin to hemolyze the RBCs. The stopper is removed from a tube and buffer is poured into each tube to the fill line $(4.0 \mathrm{mLs})$, recapped, and inverted a few times to mix the reagents. The appropriate volume of blood is added to the SickleStat tube, recapped, inverted a few times, and placed in a rack at room temperature. After a six minute incubation period, each tube is compared to a lined card to determine turbidity. Presence of turbidity indicates a positive sickle cell sample (AS or SS) and a clear solution is obtained with negative samples (AA).

Table 1. Blood volume to be used in the test method based on hematocrit value.

\begin{tabular}{cc}
\hline Hematocrit $(\%)$ & Blood Volume $(\mu \mathrm{L})$ \\
15 & 85 \\
20 & 76 \\
25 & 68 \\
30 & 60 \\
35 & 53 \\
40 & 45 \\
45 & 38 \\
50 & 30 \\
55 & 22 \\
60 & 15 \\
\hline
\end{tabular}

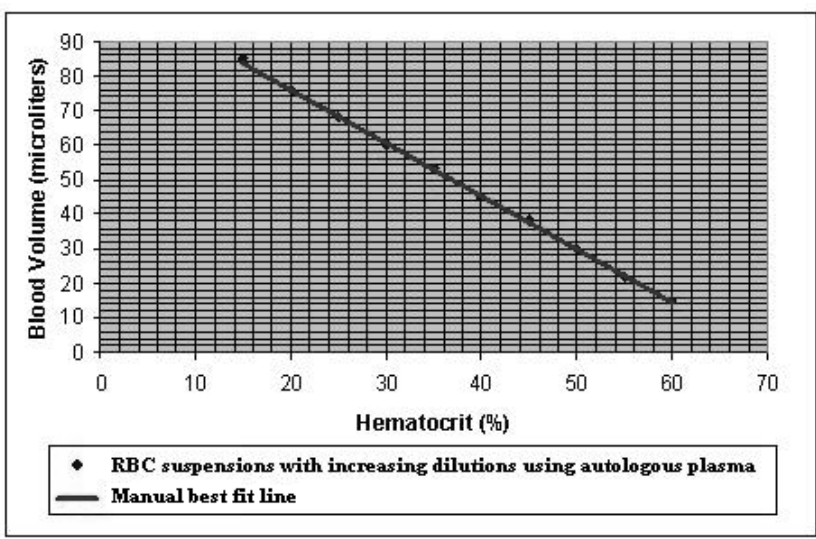

Figure 1. Blood volume adjustments based on hematocrit to achieve same absorbance reading as the $35 \%$ hematocrit standard A single blood sample was aliquoted into 10 portions, centrifuged to separate cells from plasma and remixed using different blood to cell proportions to create 10 different hematocrit readings in $5 \%$ hematocrit increments from $15 \%$ to $60 \%$. Hematocrit was performed and the blood volume needed to generate the same absorbance reading as the $35 \%$ hematocrit standard, by hemolyzing the sample in $4 \mathrm{mLs}$ of water, was determined by trial and error.

During the incubation period, filter papers were folded in half twice and placed into funnels over small $50 \mathrm{~mL}$ beakers. Following incubation the SickleStat tubes were read visually for turbidity, recorded as positive or negative and centrifuged at approximately 3500 RPMs for five minutes. The centrifuged tubes were inspected for a red or white band of material floating on top of the supernatant and the color of the supernatant is compared to a color chart provided. Each SickleStat tube is then poured onto the filter paper and allowed to filter to completion (approximately 20 minutes). The filtrate is poured from the beaker back into the cleaned $12 \times 75 \mathrm{~mm}$ glass test tube and the absorbance is measured at $540 \mathrm{~nm}$. A SickleStat tube with buffer but no blood is used as the absorbance blank. An absorbance reading is taken on the filtrate and the amount of precipitate left on the filter paper is estimated using a $0-4+$ Likert scale. The designation $\mathrm{W}+$ is used to describe precipitate in the filter paper that was present but less than $1+$ in amount. The turbidity of the original solution, color of the band of material floating after centrifugation, the color of the supernatant, absorbance of the filtrate, and amount of precipitate were recorded on the report form.

\section{Alkaline Agarose Hemoglobin Electrophoresis}

Blood samples collected in EDTA are centrifuged and 
the packed cells are washed three times in saline. Ten microliters of the packed RBCs are mixed with $100 \mu \mathrm{L}$ of the hemolysate reagent to release the hemoglobin from the cells and dilute the sample. Each blood sample and control (AFSC and $\mathrm{AA}_{2}$ ) to be tested were added to one of the ten wells in the sample application chamber and transferred to the gel. The gel is placed in the QuickGel chamber and electrophoresed for 15 minutes at 550 volts. The gel is dried, stained with acid blue stain, destained with citrate, dried again, and visually analyzed to identify bands representing $\mathrm{HbA}, \mathrm{F}, \mathrm{S}$ and C.

\section{RESULTS}

\section{Standardizing Blood Inoculum}

Figure 1 represents the blood volume needed to produce an absorbance reading equivalent to a standard blood sample with a $35 \%$ hematocrit once the RBCs are hemolyzed in $4 \mathrm{~mL}$ of water.

Table 1 is an example of a table constructed to improve the ease for the operator to determine the appropriate blood volume to be used with Sickle Confirm. Tables used in the field to perform Sickle Confirm will include a wide range of hematocrit readings in $1 \%$ hematocrit increments.

\section{Sickle Confirm Test Method}

For each of the ten positive sickle cell samples and the eight normal samples, nine data points were recorded, three for each filter paper type. These data included the final absorbance of the filtrate, flow rate in minutes, and amount of precipitate on the filter paper using a $0-4+$ Likert scale $\left(0_{+}=\right.$no precipitate, $1+=$ small amount of precipitate, $2+=$ medium amount of precipitate, $3+=$ much precipitate, $4+=$ maximum amount of precipitate). The data are represented in Table 2 .

Table 3 illustrates the absorbance readings of twenty five blood samples subjected to the new Sickle Confirm method. Samples are segregated into three groups (SS, AS, AA) based on their sickle cell zygosity as determined by alkaline hemoglobin electrophoresis. The relative amount of $\mathrm{HbS}$ precipitate on the filter paper was also estimated on a 0-4 Likert Scale. The two blank rows, between samples 7 and 8 and samples 23 and 24 , segregate the samples into the three zygosity groups, SS, AS, AA respectively.
Figure 2 illustrates the relationship of the 25 blood samples based on absorbance of the filtrate ( $\mathrm{X}$ axis), the amount of precipitate on the filter paper ( $\mathrm{Y}$ axis), and the zygosity as determined by hemoglobin electrophoresis. The circles represent each sample and the diagonal band running from the upper left to the lower right illustrates the relationship of the majority of the samples based on absorbance readings vs the amount of HbS trapped on the filter paper. The three shaded vertical bands group the samples by zygosity based on alkaline hemoglobin electrophoresis results. The open circles $(\mathrm{N}=20)$ represent samples that fit the model and the dark circles $(\mathrm{N}=5)$ represent samples that fall outside the model.

\section{DISCUSSION}

\section{Standardizing Blood Inoculum}

If absorbance of the total non-HbS is to be the primary outcome measure used to determine zygosity, then differences in the starting hematocrit may influence the final absorbance measurement to a greater extent than the non-HbS remaining in the sample following separation. To correct this problem we elected to standardize the blood inoculum introduced into the test system prior to testing.

Hematocrit was the measure selected to determine the $\mathrm{RBC}$ inoculum because it is an inexpensive and reliable method to assess oxygen carrying capacity of the blood and is commonly used in developing countries. A target hematocrit of $35 \%$ was selected because it represents the average hematocrit obtained from Haitian patients by the author during annual mass screening efforts performed in Haiti. A series of tubes were created with increasing hematocrit values and the absorbance obtained from the $35 \%$ tube became the target absorbance when $50 \mu \mathrm{L}$ of the mixture was hemolyzed in 4 milliliters of water. The $50 \mu \mathrm{L}$ of blood in $4 \mathrm{~mL}$ of water represents the blood to diluent ratio in the Chembio hemoglobin solubility test used as the basis for the new method. Water was used to completely hemolyze the RBCs to determine maximum absorbance of the solution based on the number of RBCs in the inoculum. The inoculum of all other hematocrit tubes were altered by trial and error to achieve the same absorbance as the $35 \%$ tube using an inoculum of $50 \mu \mathrm{L}$. The results appeared linear so a table was created for laboratories in developing countries to expedite the procedure and reduce errors. 
Table 2. Comparison of sickle cell positive $\&$ normal samples using filtration separation

\begin{tabular}{|c|c|c|c|c|c|c|c|c|c|}
\hline \multirow{2}{*}{$\begin{array}{l}\text { Sample } \\
S=\text { sickle } \\
N=\text { normal }\end{array}$} & \multicolumn{3}{|c|}{$\begin{array}{l}\text { P2 Filter Paper: } \\
\text { slow - fine }\end{array}$} & \multicolumn{3}{|c|}{$\begin{array}{l}\text { P5 Filter Paper: } \\
\text { slow - med }\end{array}$} & \multicolumn{3}{|c|}{$\begin{array}{l}\text { P8 Filter Paper: } \\
\text { fast - course }\end{array}$} \\
\hline & Abs & $\begin{array}{l}\text { Flow } \\
(\min )\end{array}$ & $\begin{array}{c}\text { PPT } \\
(0-4+)\end{array}$ & Abs & $\begin{array}{l}\text { Flow } \\
(\mathrm{min})\end{array}$ & $\begin{array}{c}\text { PPT } \\
(0-4+)\end{array}$ & Abs & $\begin{array}{l}\text { Flow } \\
(\mathrm{min})\end{array}$ & $\begin{array}{c}\text { PPT } \\
(0-4+)\end{array}$ \\
\hline$S+31$ & .208 & 53 & $3+$ & .202 & 9 & $3+$ & .394 & 2 & $3+$ \\
\hline$S+7$ & .271 & 62 & $2+$ & .313 & 17 & $2+$ & .488 & 9 & $2+$ \\
\hline$S+35$ & .385 & 70 & $4+$ & .313 & 20 & $4+$ & .822 & 10 & $4+$ \\
\hline$S+34$ & .509 & 64 & $3+$ & .674 & 16 & $2+$ & .695 & 8 & $1+$ \\
\hline$S+26$ & .564 & 47 & $3+$ & .686 & 11 & $3+$ & 1.050 & 9 & $2+$ \\
\hline$S+23$ & .628 & 72 & $2+$ & .651 & 36 & $2+$ & 1.209 & 7 & $2+$ \\
\hline$S+28$ & .690 & 47 & $2+$ & .663 & 13 & $2+$ & 1.048 & 4 & $1+$ \\
\hline$S+33$ & .809 & 55 & $2+$ & .628 & 16 & $2+$ & .860 & 12 & $1+$ \\
\hline$S+27$ & .814 & 47 & $3+$ & .936 & 11 & $3+$ & 1.027 & 11 & $2+$ \\
\hline$S+30$ & .925 & 50 & $2+$ & .782 & 10 & $2+$ & 1.011 & 9 & $2+$ \\
\hline MEAN & .580 & 56.7 & $2.6+$ & .585 & 15.9 & $2.5+$ & .860 & 8.1 & $2+$ \\
\hline N-18 & .609 & 48 & $0+$ & .821 & 24 & $0+$ & 1.001 & 3 & $0+$ \\
\hline N-9 & .676 & 49 & $0+$ & .920 & 12 & $0+$ & .965 & 7 & $0+$ \\
\hline N-6 & .749 & 62 & $0+$ & .885 & 26 & $0+$ & 1.024 & 2 & $0+$ \\
\hline $\mathrm{N}-19$ & .775 & 48 & $0+$ & .787 & 20 & $0+$ & .897 & 5 & $0+$ \\
\hline $\mathrm{N}-3$ & .780 & 64 & $0+$ & .942 & 14 & $0_{+}$ & 1.041 & 15 & $0+$ \\
\hline $\mathrm{N}-17$ & .813 & 50 & $0+$ & .886 & 15 & $0+$ & .864 & 9 & $0+$ \\
\hline N-8 & .814 & 49 & $0+$ & .956 & 16 & $0+$ & 1.159 & 4 & $0+$ \\
\hline $\mathrm{N}-10$ & .838 & 53 & $0+$ & 1.032 & 11 & $0_{+}$ & 1.085 & 6 & $0+$ \\
\hline MEAN & .757 & 52.9 & $0+$ & .904 & 17.3 & $0+$ & 1.001 & 6.4 & $0+$ \\
\hline
\end{tabular}

\section{Sickle Confirm Test Method}

The faster flowing filter paper (P8) has a larger pore size compared to the medium P5 paper and the slow flowing P2 paper (Table 2). In all three filter paper types used, a lower mean absorbance was produced from the sickle cell samples compared to the normal samples. However, the mean difference in absorbance for the P8 filter paper was only 0.141 creating minimal separation between the homozygous and heterozygous samples. In addition, the greatest overlap of absorbance readings between the two groups occurred with the P8 filter paper. This suggests that the pore size of the P8 paper is too large allowing $\mathrm{HbS}$ precipitate to pass through and become part of the filtrate that is measured spectrophotometrically. Of the ten positive sickle cell samples tested using the P8 filter paper, five produced an absorbance reading of greater than 1.010 which is larger than the absorbance of the lowest normal sample. Last, absorbance readings above 1.000 are approaching the upper end of the linear range for the spectrophotometer which dramatically reduces sensitivity.

The slower rate P2 filter paper produced mean absorb- ance readings of .580 for the sickle positive samples and .757 for the sickle negative samples. As with the P8 filter paper these mean absorbance values are fairly similar with a mean difference of 0.177 . Another similarity with the P8 data is that five of ten sickle positive samples filtered with P2 filter paper produced larger absorbance readings than the normal sample with the lowest absorbance. Last, the average flow rate using P2 filter paper is nearly an hour.

The medium flow rate P5 filter paper was clearly the superior method of hemoglobin separation using filtration. The P5 filter paper showed a greater mean difference in absorbance (0.319) between the sickle positive samples (.585) and the sickle negative (.904) samples. There was only one sickle positive sample that produced an absorbance greater than the lowest sickle negative sample demonstrating little overlap between groups. Last, the average flow rate was 15.9 minutes.

Based on the observation that centrifugation causes the polymerized $\mathrm{HbS}$ molecules to aggregate in a band on top of the solution, the Sickle Confirm method involves a centrifugation step followed by a filtration step to 
maximize separation. When the Sickle Confirm method was applied to 25 de-identified EDTA blood samples and their zygosity determined by hemoglobin electrophoresis, the absorbance readings effectively separated the samples into the three zygosity groups (Table 3). A natural break occurred between the homozygous and heterozygous samples denoted by the blank row between samples 7 and 8 . All the SS patients were under the care of a physician at the time of blood collection and have presumably undergone various forms of treatment. Two common forms of sickle cell treatment, blood transfusions and hydroxyuria, raise the $\mathrm{HbA}$ and $\mathrm{HbF}$ levels respectively, which artificially increases the absorbance compared to an untreated patient. Since the Sickle Confirm method is designed as a diagnostic test for untreated patients in developing countries, the cutpoint between homozygotes and heterozygotes is expected to be slightly lower (0.4-0.45) than the absorbance break in the data. Likewise, a natural break occurred between the heterozygous and normal samples \#23 and \#24. There was one positive sickle cell sample (\#23) that produced an absorbance higher than the two normal samples. This sample showed very little precipitate $\left(\mathrm{W}_{+}\right)$on the filter paper and a dense $\mathrm{HbA}$ band on electrophoresis suggesting that the patient was probably transfused prior to blood collection increasing the $\mathrm{HbA}$ concentration and decreasing the $\mathrm{HbS}$ concentration. Since true sickle cell heterozygotes rarely need treatment, this sample is likely a sickle cell homozygote that was transfused causing it to band like a heterozygote with an unusually high $\mathrm{HbA}$ level. There was another heterozygous sample (\#21)

Table 3. Absorbance readings using Sickle Confirm \& P5 filter paper vs. sickle cell zygosity.

\begin{tabular}{|c|c|c|c|c|c|}
\hline$\#$ & Sample ID & $\begin{array}{c}\text { Solubility } \\
\text { Test }\end{array}$ & $\begin{array}{l}\text { Zygosity by } \\
\text { Electrophoresis }\end{array}$ & Absorbance & $\begin{array}{l}\text { cipitiate on Filter } \\
\text { Paper }(0-4+)\end{array}$ \\
\hline 1 & CGCH24 & Positive & Homozygote & 0.246 & $4+$ \\
\hline 2 & CGCH8 & Positive & Homozygote & 0.29 & $4+$ \\
\hline 3 & SLUH4 & Positive & Homozygote & 0.321 & $4+$ \\
\hline 4 & CGCH10 & Positive & Homozygote & 0.36 & $4+$ \\
\hline 5 & SLUH20 & Positive & Homozygote & 0.327 & $3+$ \\
\hline 6 & SLUH12 & Positive & Homozygote & 0.454 & $3+$ \\
\hline 7 & SLU21 & Positive & Homozygote & 0.456 & $3+$ \\
\hline 8 & SLUH19 & Positive & Heterozygote & 0.602 & $2+$ \\
\hline 9 & SLUH3 & Positive & Heterozygote & 0.623 & $2+$ \\
\hline 10 & SLUH17 & Positive & Heterozygote & 0.657 & $1+$ \\
\hline 11 & CG2 & Positive & Heterozygous & 0.658 & $2+$ \\
\hline 12 & CG1 & Positive & Heterozygote & 0.67 & $3+$ \\
\hline 8 & SLUH19 & Positive & Heterozygote & 0.602 & $2+$ \\
\hline 9 & SLUH3 & Positive & Heterozygote & 0.623 & $2+$ \\
\hline 10 & SLUH17 & Positive & Heterozygote & 0.657 & $1+$ \\
\hline 11 & CG2 & Positive & Heterozygous & 0.658 & $2+$ \\
\hline 12 & CG1 & Positive & Heterozygote & 0.67 & $3+$ \\
\hline 13 & SLUH11 & Positive & Heterozygote & 0.691 & $1+$ \\
\hline 14 & SLUH6 & Positive & Heterozygote & 0.696 & $2+$ \\
\hline 15 & CGCH1 & Positive & Heterozygote & 0.731 & $4+$ \\
\hline 16 & CGCH16 & Positive & Heterozygote & 0.751 & $3+$ \\
\hline 17 & CHGH2 & Positive & Heterozygote & 0.783 & $3+$ \\
\hline 18 & SLUH5 & Positive & Heterozygote & 0.785 & $2+$ \\
\hline 19 & SLUH9 & Positive & Heterozygote & 0.785 & $1+$ \\
\hline 20 & SLUH25 & Positive & Heterozygote & 0.805 & $1+$ \\
\hline 21 & SLUH22 & Positive & Heterozygote & 0.815 & $\mathrm{~W}_{+}$ \\
\hline 22 & SLUH13 & Positive & Heterozygote & 0.899 & $1+$ \\
\hline 23 & $\mathrm{CGCH} 23$ & Positive & Heterozygote & 0.981 & $\mathrm{~W}_{+}$ \\
\hline 24 & Researcher & Negative & Normal & 0.946 & $0+$ \\
\hline 25 & Researcher & Negative & Normal & 0.966 & $0+$ \\
\hline
\end{tabular}


with a weak $\left(\mathrm{W}_{+}\right)$amount of precipitate and three samples (\#19, \#20 and \#22) with a $1+$ amount of precipitate. Again, since these samples likely represent recently treated patients, the absorbance cutoff between heterozygotes and normal samples is likely to be closer to 0.8 if not lower in undiagnosed, untreated patients.

Figure 2 illustrates the relationship between the amount of precipitate trapped in the filter paper $(\mathrm{HbS})$ and the absorbance of the filtrate $\left(\mathrm{HbA}+\mathrm{HbA}_{2}+\mathrm{HbF}\right)$ in the context of the three zygosity groups as defined by hemoglobin electrophoresis. As can be seen, 20 of 25 samples fit the model perfectly even though the absorbance readings are likely to be skewed upward resulting from treatment in the form of transfusion, hydroxyuria, or both. The diagonal band running from the upper left to the lower right corner of the figure represents the relationship between the amount of $\mathrm{HbS}$ precipitate and non-HbS in the filtrate.

Four of the five outliers were heterozygous samples that plotted above the diagonal band. These samples showed more precipitate $(3+-4+)$ but produced an absorbance reading in the heterozygous range, corresponding with the true zygosity as determined by hemoglobin electrophoresis. This finding can be explained in two ways. First, the measure of precipitate on the filter paper is a subjective measure and therefore less accurate and reproducible. Second, it seems reasonable that the $\mathrm{HbS}$ molecules within the RBCs of a treated homozygote could have begun the process of polymerization prior to hemolysing in the testing reagent reducing the polymerization interference from the $\mathrm{HbA}$ molecules in the normal transfused cells. This could improve polymerization efficiency and maximize $\mathrm{HbS}$ trapping while still introducing significant amounts of $\mathrm{HbA}$ from the transfused RBCs.

The remaining outlier was the heterozygous sample that had an absorbance slightly higher than the two normal samples. Again, this is likely the result of a recent transfusion in a very anemic sickle cell homozygote that dramatically diluted the patient's HbS containing RBCs leaving only enough $\mathrm{HbS}$ to produce a small amount of precipitate on the filter paper $\left(\mathrm{W}_{+}\right)$.

In summary, the Sickle Confirm method can effectively separate homozygote, heterozygote and normal samples based on a series of observations to include the turbidity

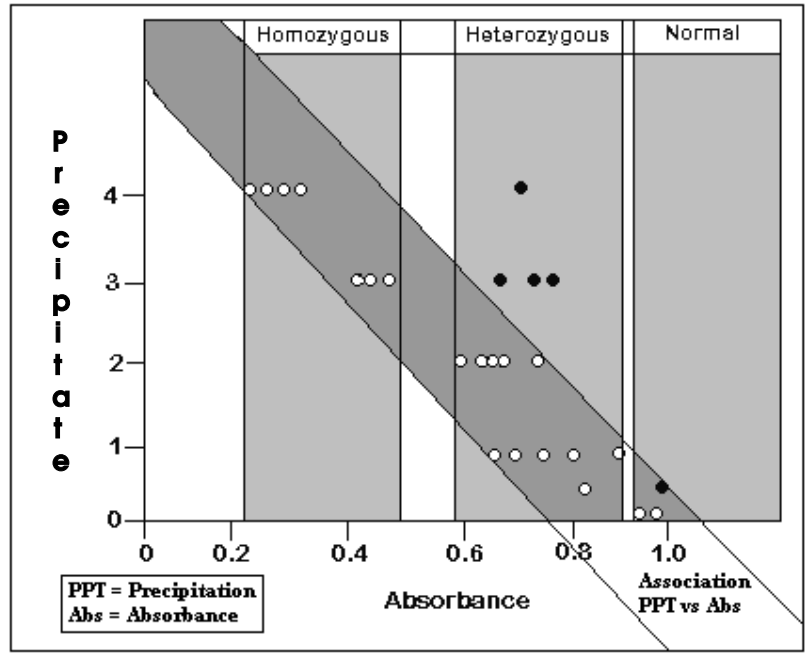

Figure 2. Association between absorbance and the amount of precipitate on the filter paper. Twenty five blood samples, 7 homozygous for sickle cell (SS), 16 heterozygous for sickle cell (AS) and 2 normal samples (AA) were tested using Sickle Confirm. All 25 samples were segregated into the three zygosity categories, as determined by electrophoresis, and the absorbance of the filtrates were plotted against the amount of precipitate on the filter paper. Open circles represent samples that fit the model and dark circles represent samples that fell outside the model.

of the sample, color of the floating band, color of the supernatant when compared to a color standard, precipitate on the filter paper, and the quantitative measure of filtrate absorbance. The original turbidity of the samples along with the color of the band of material floating on the supernatant following centrifugation was able to differentiate normal AA samples (no turbidity and white band) from AS samples (turbidity and red band) for every sample. Using absorbance alone there were no overlapping samples between the homozygotes and heterozygotes producing a perfect sensitivity and specificity when differentiating these samples. There was one heterozygous sample with an absorbance reading slightly higher than the normal samples but was likely to be a recently transfused patient and the test is designed for untreated patients seeking an initial diagnosis. As indicated above, heterozygous and normal samples would be differentiated early in the testing process based on the initial presence or absence of turbidity in the solution and the presence or absence of a red band on the surface of the solution following centrifugation making the determination of normal samples by absorbance readings unnecessary. Using the initial turbidity reading and the color of the floating 
band, the sensitivity and specificity of the Sickle Confirm method to distinguish sickle cell heterozygotes from normal AA samples was also $100 \%$ for both.

\section{Future Studies}

Field testing studies have commenced in Haiti where the Sickle Confirm method is being used to screen untreated Haitian patients for sickle cell and to determine zygosity as confirmed by hemoglobin electrophoresis. Comparative studies by the author have also commenced where the diagnostic efficiency of the Sickle Confirm method is being studied on Haitian patients when performed by Haitian laboratory professionals. Since the ultimate goal is to develop comprehensive sickle cell centers in underdeveloped countries, we are also developing other lab testing procedures to support a sickle cell treatment program to include a hemoglobin assay not requiring electricity or battery power and $\mathrm{HbF}$ and $\mathrm{HbC}$ assays.

\section{REFERENCES}

1. Herrick JB. Peculiar elongated and sickle-shaped red blood corpuscles in a case of severe anemia. Arch Intern Med. 1910;6:517-21.

2. Pauling, L., H. A. Itano, S. J. Singer, and I. C. Wells. Sickle cell anemia, a molecular disease. Science. 1949;110:543-8.

3 Ingram, V. M. Gene mutations in human hemoglobin: the chemical difference between normal and sickle cell hemoglobin. Nature. 1957;180:326-8.
4. Diallo D. Tchernia G. Sickle cell disease in Africa. Curr Opin Hematol. 2002;9(2):111-6.

5. Randolph TR. Prevalence of sickle cell anemia in Northern Haiti. CLS. 2010;23(2):79-83.

6. Randolph, TR. In: Hemoglobinopathies from Clincial Hematology and Fundamentals etc., Rodak, Doig, Fritzma (eds),2007.

7. Ohene-Frempong K, Weiner SJ, Sleeper LA, et al. Cerebrovascular accidents in sickle cell disease: rates and risk factors. Blood 1998;91:288-94.

8. Armstrong FD, Thompson RJ, Wang W, et al. Cognitive functioning and brain magnetic resonance imaging in children with sickle cell disease. Neurophysiology Committee of the Cooperative Study of Sickle Cell Disease. Pediatrics 1996;97:864-70.

9. Beutler E: The sickle cell diseases and related disorders. In Beutler E, Lichtman MA, Coller BS, et al (eds): Williams Hematology, 6th ed. New York: McGraw-Hill, 2001.

10. Preboth, M: Management of pain in sickle cell disease. Am Fam Phy 2000;61(5):1-7.

11. Huntsman RG, Barclay GPT, Canning DM and Yawson GI. A rapid whole blood solubility test to differentiate the sickle-cell trait from sickle-cell anaemia. J Clin Path 1970;23:781-3.

12. Serjeant BE and Serjeant GR. A whole blood solubility and centrifugation test for sickle cell hemoglobin. Am J Clin Pathol 1971;58:11-3.

13. Bowie LJ and Dohnal JC. Proportion of hemoglobin $S$ in blood, as determined from solubility measurements. Clin Chem 1983;29(2):325-8.

14. Scabet M, McRoyan C, Liu G, et al. A rapid assessment of percentage hemoglobin-S in whole blood. Am J Clin Pathol 1986;86:759-61.

\section{Clinical Laboratory Science Online}

Need to check the journal online? Scan here with

your QR scanner on your smartphone or tablet. 